\title{
The Diagnosis of Periodontal Disease in Private Practice
}

\author{
D.J. Apsey, ${ }^{*}$ N. Kaciroti, ${ }^{\dagger}$ and W.J. Loesche ${ }^{\ddagger}$
}

Background: Periodontal disease is an inflammatory reaction to the bacteria in dental plaque. The present study compared the prevalence of periodontal disease in patients using as a diagnostic either probing depth measurements, an inflammatory marker such as numbers of white blood cells in plaque samples, or microbiological markers such as the microscopic count and the benzoyl-DL-arginine naphthylamide (BANA) test.

Methods: Teeth with the most inflammation and/or deepest pockets in each quadrant were probed and subgingival plaque was sampled from 1,043 consecutive new patients enrolled in a private practice. Multivariate "diagnostic" models were developed based upon the probing depth (general linear models), percentage of white blood cell-positive and percentage of BANA-positive plaques (logistic regression models) to determine the prevalence of patients with periodontal disease.

Results: Plaque samples were removed from 3,694 sites. Fifty-two percent of sampled pockets were $>4 \mathrm{~mm} ; 49 \%$ of sites were inflamed, using the presence of white blood cells, and $28 \%$ were infected using the BANA test. Diagnostic models were highly significant at $P<0.0001$. The white blood cell model was the most parsimonious as demonstrated by the lowest Akaike information criteria statistic and had the highest receiver operator characteristic (ROC) curve relative to the probing depth and BANA models.

Conclusions: Periodontal disease can be diagnosed chairside by the presence of white blood cells in plaque samples, a finding that reflects the inflammatory nature of the disease process. This approach would reduce the misclassification of subjects as having periodontal disease (130 patients in the present study who had pockets) but minimal evidence of an inflammatory response. J Periodontol 2006;77:1572-1581.

\section{KEY WORDS}

BANA; diagnosis; periodontal diseases; white blood cells.

\footnotetext{
* Private practice, Fraser, MI

$\dagger$ Center for Human Growth and Development, University of Michigan, Ann Arbor, MI

$\ddagger$ Department of Biological and Materials Sciences, School of Dentistry, University of Michigan.
}

$\mathrm{P}$ eriodontal disease is a common affliction of adults with about $50 \%$ having gingivitis and 30\% having periodontitis. ${ }^{1-3}$ Most forms reflect a tissue inflammatory response to bacterial accumulations on the teeth. These accumulations represent an overgrowth of a finite number of bacterial species, mostly anaerobes, in the subgingival dental plaque, thereby making periodontal disease a chronic but specific bacterial infection. ${ }^{4}$ The American Academy of Periodontology 5 states that "Periodontal (gum) diseases, including gingivitis and periodontitis, are serious infections that, left untreated, can lead to tooth loss." Most periodontology textbooks define periodontal disease as an inflammatory reaction to a microbial infection associated with dental plaque that results in tissue loss. ${ }^{6,7}$ For many patients, the clinical recognition of periodontal disease is made by probing measurements about the teeth and by radiographs, which document the amount of tissue support that has been lost about the tooth. Bleeding on probing is commonly found in periodontal disease but has a low sensitivity as a predictor of periodontal disease progression because of a high frequency of false-positive (FP) responses. ${ }^{8}$

The absence of microbiological diagnostic criteria in clinical periodontics has some practical basis as the culturing of plaque samples for anaerobes is fraught with technical problems and results can take weeks to be determined. Likewise, the taking of blood or gingival samples

doi: 10.1902/jop.2006.050449 
for measurements of inflammatory markers is not consistent with dental practice. But these objections do not apply to the chairside microscopic examination of plaque samples, which, since Leuwenhoek in the 16th century, have shown the abundance of motile organisms, such as spirochetes, in samples removed from disease sites. ${ }^{9,10}$ Keyes and Rams ${ }^{11}$ demonstrated that the microscopic examination of plaque samples could be used to adjust the treatment regimens for individual patients by monitoring the numbers of white blood cells (WBCs) and motile organisms. Successful treatment(s) resulted in a disappearance of WBCs and a reduction in motile organisms associated with an increase in cocci and rods.

Bacteriological studies have consistently shown the dominance of a limited number of anaerobic species such as Treponema denticola, Porphyromonas gingivalis, and Tannerella forsy thensis in plaques removed from disease sites. ${ }^{4,12,13}$ These organisms are difficult to culture but can be detected using a laboratory-based DNA technique. ${ }^{14}$ They are unique among cultivable plaque species in possessing one or more enzymes that can hydrolyze the synthetic trypsin substrate, benzoyl-DL-arginine naphthylamide (BANA). ${ }^{15}$ This enzyme(s) can be detected in plaque samples within minutes at chairside with a sensitivity and specificity comparable to DNA probes. ${ }^{16,17}$

The purpose of the present study was to compare the prevalence of periodontal disease in patients seen in a private practice using either probing depth measurements or an inflammatory marker such as numbers of WBCs in plaque samples or microbiological markers such as the microscopic count and the BANA test. To our knowledge, no one has used all three approaches simultaneously in the same subjects. The findings obtained on 1,043 subjects permitted multivariate modeling to determine which of these diagnostic procedures appeared to be the most reliable in recognizing periodontal disease.

\section{MATERIALS AND METHODS}

\section{Subjects}

Data were obtained from 1,043 consecutive new dental patients enrolled between April 1997 and November 2001 at a private practice located in southeast Michigan. The practice was unusual in that it advertised an offering of non-surgical periodontal therapy, so that the patient profile was enriched by patients seeking periodontal treatment. Patients were told that most forms of periodontal disease are treatable infections and that microbiological criteria, such as a microscopic examination of plaque samples and an enzyme assay for plaque bacteria, would be routinely performed to assist in the diagnosis and monitoring of treatment results in addition to the traditional pocket probing measurements. All adults were screened on their first visit. They were aware that data from their examination would be used in a research study and that no person would be identified in any publication. Only patients who agreed that their data could be used were included in the analysis. The procedures endorsed by the Helsinki Declaration were followed. The personal identifiers of the patients were rendered in a cryptic form in the file used in the statistical analysis and were never accessed.

\section{Selection of Teeth and Sampling Procedures}

Teeth with the most inflammation (tissue swelling, bleeding, and suppuration) and/or deepest pockets in each quadrant were probed, and subgingival plaque was removed and sampled for microscopic and enzyme markers. As some patients had no obvious clinical disease, this meant that periodontally healthy teeth and diseased teeth were sampled. The probing depth(s) and gingival recession of selected teeth were determined using a Michigan-type probe. A subgingival plaque sample was obtained with a Columbia 13/14 curet from an interproximal site on a tooth. Approximately half of this sample was added to a drop of saline on a microscopic slide, and the remainder was applied to the lower reagent matrix of the BANA test. About 10 to 20 seconds after the sample was collected, the papillary bleeding score (PBS) was measured. ${ }^{18}$

\section{Microscopic Examination}

Microscopic samples were read immediately using 400x magnification on a phase-contrast microscope. $\S$ From one to five microscopic fields were examined on each sample for the presence of WBCs, motile organisms (spirochetes, spinning rods, motile cocci, and gliding rods), cocci, spiral rods, amoeba, and trichomonads. Levels of each microscopic category were scored as follows: $0=$ not present in any of the five microscopic fields; 1 = few present in one or more fields; 2 = present in every field in moderate numbers; and 3 = present in high numbers. ${ }^{11}$ For the statistical analysis, each site was scored either as a 0 (no infection; microscopic score of 0 or 1 ) or 1 (infection; microscopic score of 2 or 3 ) for each morphotype.

\section{BANA Test}

The plaque on the scaler was wiped onto the BANAimpregnated strip found at the bottom of a reagent card. I The upper reagent strip was activated by lightly moistening it with distilled water. The two strips were folded to contact each other, and the card was inserted into a heating block and incubated for 5 minutes at $55^{\circ} \mathrm{C}$. ${ }^{15}$ Naphthylamide released due to the presence of BANA-hydrolyzing bacterial species diffused into

$\S$ Meiji Microscope, Oratec, Manassas, VA.

\| Oratec. 
the upper reagent strip where it reacted with a dye to form a permanent blue color. A score of 1 was given when no blue color was visible, a score of 2 when a faint blue color was noted, and a score of 3 when a distinct blue color was observed. For statistical analysis, scores of 2 and 3 were grouped as indicative of a positive response. Each site was then scored either as a 0 (no infection; BANA score of 1) or 1 (infection; BANA score of 2 or 3 ).

\section{PBS}

The amount of bleeding present was scored using the PBS, ${ }^{18}$ a six-level scale with a score of 0 reflecting health, 1 indicating some degree of inflammation in the absence of bleeding, 2 indicating spotting of blood in the papilla, and PBS from 3 to 5 corresponding to various degrees of bleeding. The same investigator (DJA) collected all plaque samples and judged each PBS.

\section{Statistical Analysis}

The data were entered in a spreadsheet program, " and batch-transferred to statistical software." Data for the sampled teeth were averaged so that only per patient values were used in the statistical analysis. For the microscopic and BANA data, both average values and percentage of infected sites were used, i.e., percentage of sites with a 1 (infected) score for the variable under consideration. Descriptive statistics, correlations, and $\chi^{2}$ frequency distributions were determined for variables of interest. The following models were developed: three general linear models with probing depths $\geq 4, \geq 5$, and $\geq 6 \mathrm{~mm}$ as the primary outcome and four multiple logistic regression models in which the presence of an infection was determined by the number of subjects with $>0 \%$ of sites showing an infection with WBCs; $>50 \%$ of sites showing an infection with WBCs; $>0 \%$ of sites showing an infection with a BANA-positive score; and $>50 \%$ of sites showing an infection with BANA-positive sites. The predictor variables were age, gender, smoking status (never, quit, and current), PBS, gingival recession, microscopic morphotypes, and, where appropriate, probing depth, BANA score, and WBC score. Receiver operating characteristic (ROC) curves (Fig. 1), a global assessment of explanatory ability created by plotting sensitivity by 1 -specificity, were constructed for the various models using probing depth first and then the percentage of WBCs or percentage of BANA as the diagnostic criterion. This provided an estimate of the correct identification of treatment needs versus FP identification for each of the diagnostic criteria.

\section{RESULTS}

The participants were $53 \%$ male, averaged 46.9 years of age, and $23 \%$ were current smokers, $17 \%$ were former smokers, and $60 \%$ had never smoked. Plaque

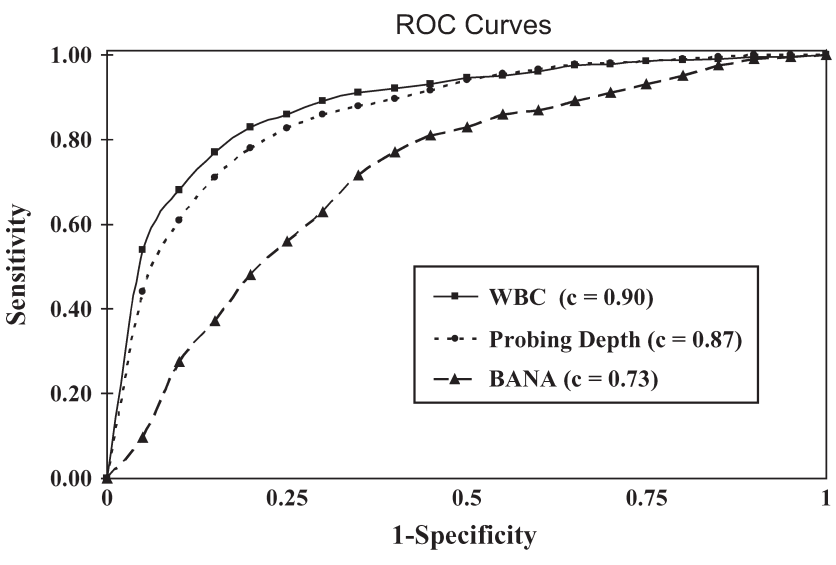

Figure I.

ROC curves for WBC, probing depth, and BANA models

samples were removed from between two and eight sites per patient ( $72 \%$ provided four sites) for a total of 3,694 sites. The selection of sites was based on clinical inflammation and emphasized molars $(\mathrm{N}=2,534$ sites; $68.6 \%$ ) over bicuspids ( $N=701$ sites; $19.0 \%)$ and anterior teeth ( $\mathrm{N}=459$ sites; $12.4 \%)$. The average probing depth was $4.6 \mathrm{~mm}$, and $52 \%$ of the sampled pockets were $>4 \mathrm{~mm}$. Forty-nine percent of the sampled sites were inflamed, using the presence of WBCs as the diagnostic criteria, and $28 \%$ were infected with anaerobic species using the BANA test (Table 1).

The current and former smokers were combined as an ever-smoked group and compared to the neversmoked group (Table 1). Smokers were significantly younger (45.0 versus 48.2 years). Pockets were 0.6 $\mathrm{mm}$ deeper, and attachment loss was $0.9 \mathrm{~mm}$ greater $(P<0.001)$ in smokers compared to non-smokers. Sixty-five percent of the sampled pockets were $>4$ $\mathrm{mm}$ in the smokers compared to $46 \%$ in the neversmoked group. Smokers had significantly higher percentages of sites that were WBC and BANA positive and were significantly more likely to score positive on most microbiological parameters, i.e., spirochetes (35\% versus $21.6 \%$ ), small gliding rods ( $34 \%$ versus $24 \%$ ), and spinning rods (48\% versus $38 \%$ ) (Table 1 ).

Patients are usually diagnosed as having periodontal disease based upon measurements of probing depth. We were interested in how many and what types of individuals would be diagnosed if criteria indicative of an inflammatory or infectious process were used, i.e., the percentages of tooth sites that were WBC positive or which were overgrown with the BANA species. In this regard, we applied relaxed and stringent thresholds of diagnostic criteria and, as expected, observed more patients diagnosed when

II Excel, Microsoft, Redmond, WA

\# SAS version 8.2, SAS Institute, Cary, NC. 
Table I.

Demographic, Clinical, and Microbiological Characteristics of Patients Enrolled in the Study and Differences Between Smokers and Non-Smokers

\begin{tabular}{|c|c|c|c|c|}
\hline & $\begin{array}{c}\text { Total }(N=1,043) \\
\quad(\text { mean } \pm \text { SD })\end{array}$ & $\begin{array}{l}\text { Ever (current and quit) } \\
\text { Smoked }(n=4 \mid 4)\end{array}$ & $\begin{array}{l}\text { Never Smoked } \\
\quad(n=629)\end{array}$ & $\begin{array}{c}\text { Probability ( } t \text { test): Ever Versus } \\
\text { Never Smoked }\end{array}$ \\
\hline \multicolumn{5}{|l|}{ Demographics } \\
\hline Age (years) (mean $\pm S D)$ & $46.9 \pm 13.6$ & $45.3 \pm 11.5$ & $48.0 \pm 14.7$ & 0.0015 \\
\hline Gender (\% male) & 53.7 & $50 \pm 50$ & $56 \pm 50$ & 0.052 \\
\hline Smokers (\%) & 39.7 & 100 & 0 & \\
\hline \multicolumn{5}{|l|}{ Clinical measurements } \\
\hline PBS & $2.0 \pm 1.1$ & $2.0 \pm 1.0$ & $2.0 \pm 1.2$ & Not significant \\
\hline Probing depth $(\mathrm{mm})$ & $4.6 \pm 1.5$ & $4.9 \pm 1.5$ & $4.3 \pm 1.5$ & $<0.00$ \\
\hline Pockets $>3$ mm (\%) & $78 \pm 41$ & $87 \pm 33.7$ & $72 \pm 45$ & $<0.00$ । \\
\hline Pockets >4 mm (\%) & $52 \pm 50$ & $65 \pm 48$ & $46 \pm 50$ & $<0.00 \mid$ \\
\hline Pockets >5 mm (\%) & $31 \pm 46$ & $38 \pm 49$ & $26 \pm 44$ & $<0.001$ \\
\hline Recession $(\mathrm{mm})$ & $0.6 \pm 0.9$ & $0.8 \pm 0.9$ & $0.5 \pm 0.8$ & $<0.001$ \\
\hline Attachment level (mm) & $5.1 \pm 1.9$ & $5.7 \pm 1.9$ & $4.8 \pm 1.8$ & $<0.001$ \\
\hline \multicolumn{5}{|l|}{ Microbiological tests } \\
\hline BANA positive (\%) & $28 \pm 38.4$ & $33 \pm 40$ & $25.0 \pm 37$ & 0.001 \\
\hline WBC positive (\%) & $48.8 \pm 42.4$ & $53 \pm 42$ & $46 \pm 37$ & 0.008 \\
\hline Spirochetes (\%) & $26.5 \pm 35.9$ & $35 \pm 33$ & $21 \pm 36$ & $<0.001$ \\
\hline Small (\%) & $6.1 \pm 18.1$ & $9 \pm 22$ & $4 \pm 15$ & $<0.001$ \\
\hline Large (\%) & $20.8 \pm 34$ & $26 \pm 37$ & $17 \pm 31$ & $<0.001$ \\
\hline Small gliding rods (\%) & $28 \pm 35.8$ & $34 \pm 38$ & $24 \pm 33$ & $<0.001$ \\
\hline Large gliding rods (\%) & $14 \pm 26.9$ & $18 \pm 30$ & $12 \pm 24$ & 0.004 \\
\hline Spinning rods (\%) & $41.9 \pm 39.8$ & $48 \pm 40$ & $38 \pm 39$ & $<0.001$ \\
\hline Spiral rods (\%) & $0.9 \pm 6.7$ & $1.2 \pm 7$ & $0.8 \pm 0.6$ & Not significant \\
\hline Motile cocci (\%) & $7.9 \pm 21.9$ & $9 \pm 23$ & $7 \pm 21$ & Not significant \\
\hline Protozoans (\%) & $<0.05$ & & & \\
\hline
\end{tabular}

thresholds were set low. When we used the presence of sites with probing depths $\geq 4 \mathrm{~mm}$ (relaxed) as the threshold, $78 \%$ of subjects would have been diagnosed as having periodontal disease (Table 2). When a higher (stringent) level was used, i.e., probing depths $\geq 6 \mathrm{~mm}, 31 \%$ of subjects would have been recommended for treatment. When $\geq 5 \mathrm{~mm}$ was used, as is common in clinical practice, $52 \%$ of the patients would have been diagnosed with periodontal disease.

Sixty-five percent of the subjects would have been diagnosed with periodontal disease if the presence of any site positive for WBCs was used (relaxed) compared to $54 \%$ if subjects had to have $>50 \%$ of sites that were WBC positive (stringent) (Table 2). For the BANA test, the respective values were $43 \%$ (relaxed) and $24 \%$ (stringent). The stringent WBC model $(\mathrm{N}=$ 563) and the standard probing depth model $(N=$ 540) were the most similar in the number of patients identified as having periodontal disease. The least number of patients was identified by the stringent BANA model $(N=252)$ and the stringent probing depth model $(\mathrm{N}=318)$.
We conducted a series of $\chi^{2}$ analyses in which all possible combinations of thresholds for probing depth, percentage of WBC-positive sites, and percentage of BANA-positive sites were compared to each other (Table 3). All comparisons were highly significant $(P<0.001)$, indicating that the various diagnostic criteria were mostly identifying the same patients. The Cohen statistic, which provides the к соefficient of agreement between the various diagnostic criteria, had the highest value, i.e., 0.47 , when $\leq 4$ versus $\geq 5 \mathrm{~mm}$ probing depth (reference standard) were compared to $\leq 50 \%$ of sites that were WBC positive versus $>50 \%$ of sites that were WBC positive (test), indicating that this was the best of the comparisons.

If this comparison is examined in detail (Table 4), when probing depth is used as the reference standard and WBCs are used as the test, the accuracy was $73.8 \%$ (true positive [TP] + true negative [TN]/sample size $\times$ $100)$, the sensitivity (TP/TP + false negative [FN]) was $76.4 \%$, the specificity (TN/TN + FP) was $71 \%$, the positive-predictive value (TP/TP + FP) was 
Table 2.

\section{Diagnostic Criteria Used to Assign Patients to Periodontal Treatment}

\begin{tabular}{llcc}
\hline & & $\begin{array}{c}\text { Patients Recommended } \\
\text { for Treatment }\end{array}$ \\
\cline { 3 - 4 } Diagnostic Criteria & Threshold & $\%$ & $\mathrm{~N}$ \\
\hline $\begin{array}{l}\text { Probing depth } \\
\text { Sites } \geq 4 \mathrm{~mm}\end{array}$ & Relaxed & 78.1 & 815 \\
$\quad$ Sites $\geq 5 \mathrm{~mm}$ & Standard & 52.3 & 540 \\
$\quad$ Sites $\geq 6 \mathrm{~mm}$ & Stringent & 30.9 & 318 \\
Sites in which WBCs & & & \\
are present & & & \\
$>0 \%$ & Relaxed & 65.2 & 680 \\
$>50 \%$ & Stringent & 54.0 & 563 \\
Sites in which BANA & & & \\
test is positive & & & 445 \\
$>0 \%$ & Relaxed & 42.7 & 229 \\
$>50 \%$ & Stringent & 24.2 & \\
\hline
\end{tabular}

$74.6 \%$, and the negative predictive value (TN/TN+FN) was $72.9 \%$. However, because of the similarity of sensitivity, specificity, and positive- and negativepredictive values, there would be little change in the number of subjects incorrectly diagnosed if the percentage of WBCs was used as the primary reference. For example, 550 subjects would be recommended for treatment based on the presence of probing depths $\geq 5 \mathrm{~mm}$, and 563 subjects would be recommended for treatment based upon the presence of $>50 \%$ of the sites being positive for WBCs. About $75 \%$ of these would be the same subject. Among the subjects for whom treatment is not recommended, about $72 \%$ would be the same subject.

The real differences between the two diagnostic criteria would be subjects misclassified as needing or not needing treatment. The probing depth model would recommend treatment for 130 subjects who have pockets $\geq 5 \mathrm{~mm}$ but minimal evidence of inflammatory disease and would not recommend treatment for 143 subjects who have pockets $\leq 4 \mathrm{~mm}$ but with more than $50 \%$ of sites showing clinical inflammation. The WBC model would recommend just the opposite, i.e., no treatment for the 130 subjects with pockets $\geq 5 \mathrm{~mm}$ but minimal evidence of inflammatory disease and treatment for the 143 subjects with shallow pockets but evidence of inflammation.

Patients are usually informed that they have periodontal disease based upon the presence of 5- and 6 -mm pockets. This traditional approach has rarely been validated against the presence of inflammatory markers or the presence of an infection. As explana- tory variables for all three diagnostic approaches were available for the 1,043 patients, multivariate diagnostic models were constructed using probing depths, inflammatory markers (WBCs), or an anaerobic infection (BANA scores) as the basis for diagnosing periodontal disease. Explanatory variables described in Table 1 were entered into these models to determine which of them would contribute to the diagnosis.

We looked at those probing depth, WBC, and BANA models that identified about the same number of subjects, i.e., $43 \%$ to $54 \%$, as having periodontal disease. We chose $\geq 5 \mathrm{~mm}$ as the probing depth model, as it reflects clinical practice and because it identified 540 subjects as having periodontal disease. The model of $>50 \%$ of sites positive for WBCs was chosen because it identified 563 subjects, and the model of $>0 \%$ of sites positive for BANA was chosen because it identified 445 subjects. All models were highly significant $(P$ $<0.0001$ ), but the WBC model was the most parsimonious, i.e., had the lowest Akaike information criteria (AIC) value, and exhibited the highest ROC curve (Fig. 1), indicating that it was the best of the three models. The probing depth model had a higher AIC value and lower ROC scores, whereas the BANA model had the highest AIC value and the lowest ROC scores (Table 5).

Age and smoking status were initially included in all models because of their accepted role as risk factors in periodontal disease. Age, although a weak but significant predictor of periodontal disease in the probing depth and BANA models, was not a factor in the WBC model. Likewise smoking status, although a strong predictor of periodontal disease in the probing depth model, was not a significant predictor of diagnosis in the WBC model, and "quit smoking" was a marginally significant predictor in the BANA model. In the probing depth model, the PBS, percentage of BANApositive sites, the percentage of WBC-positive sites, and the percentage of spirochetes were significant predictors of periodontal disease (Table 5). Of these variables, subjects with BANA-positive sites were 5.3 times more likely to be diagnosed with periodontal disease than subjects with no BANA-infected sites.

In the WBC model, the probing depth; PBS; percentage of BANA-positive sites; percentage of spirochetes; and two microscopic findings, the percentage of small gliding rods and the percentage of spinning rods, were significant predictors of periodontal disease. Of these variables, the percentage of small gliding rods and percentage of spinning rods had the highest odds ratios (ORs) at 6.3 and 4.9 , respectively. In the BANA model, the probing depth and percentage of WBC-positive sites were strong predictors of periodontal disease. Subjects infected with a percentage of spinning rods were 1.7 times more likely to have a diagnosis of periodontal disease. 
Table 3.

\section{Chi-Square Comparisons of Relaxed and Stringent Levels of Probing Depths, Percentage of WBC-Positive Sites, and Percentage of BANA-Positive Sites*}

\begin{tabular}{|c|c|c|c|c|c|c|c|}
\hline $\begin{array}{l}\text { Reference } \\
\text { Probing Depth }\end{array}$ & Test WBC & Accuracy (\%) & $\mathrm{FP}(\%)$ & FN (\%) & TP $(\%)$ & TN (\%) & Cohen \\
\hline$\leq 3$ versus $\geq 4 \mathrm{~mm}$ & $0 \%$ versus $>0 \%$ & 74.6 & 6.2 & 19.2 & 59 & 15.6 & 0.39 \\
\hline$\leq 4$ versus $\geq 5 \mathrm{~mm}$ & $0 \%$ versus $>0 \%$ & 70.9 & 20.8 & 8.3 & 44 & 26.5 & 0.41 \\
\hline$\leq 5$ versus $\geq 6 \mathrm{~mm}$ & $0 \%$ versus $>0 \%$ & 60.1 & 37.1 & 2.8 & 28 & 32 & 0.29 \\
\hline$\leq 3$ versus $\geq 4 \mathrm{~mm}$ & $\leq 50 \%$ versus $>50 \%$ & 68 & 4 & 28 & 50 & 18 & 0.33 \\
\hline$\leq 4$ versus $\geq 5 \mathrm{~mm}^{*}$ & $\leq 50 \%$ versus $>50 \%$ & $73.8 *$ & $14 *$ & $13 *$ & $40 *$ & $34 *$ & $0.47 *$ \\
\hline$\leq 5$ versus $\geq 6 \mathrm{~mm}$ & $\leq 50 \%$ versus $>50 \%$ & 68.6 & 27 & 4 & 27 & 42 & 0.39 \\
\hline $\begin{array}{l}\text { Reference } \\
\text { Probing Depth }\end{array}$ & Test BANA & Accuracy (\%) & FP (\%) & FN (\%) & TP (\%) & TN (\%) & Cohen \\
\hline$\leq 3$ versus $\geq 4 \mathrm{~mm}$ & $0 \%$ versus $>0 \%$ & 57.6 & 4 & 39 & 39 & 18 & 0.22 \\
\hline$\leq 4$ versus $\geq 5 \mathrm{~mm}$ & $0 \%$ versus $>0 \%$ & 67.5 & 11 & 21 & 31 & 36 & 0.36 \\
\hline$\leq 5$ versus $\geq 6 \mathrm{~mm}$ & $0 \%$ versus $>0 \%$ & 66.9 & 22 & 11 & 20 & 47 & 0.30 \\
\hline$\leq 3$ versus $\geq 4 \mathrm{~mm}$ & $\leq 50 \%$ versus $>50 \%$ & 43.3 & । & 55 & 23 & 21 & 0.12 \\
\hline$\leq 4$ versus $\geq 5 \mathrm{~mm}$ & $\leq 50 \%$ versus $>50 \%$ & 64 & 4 & 32 & 20 & 43 & 0.30 \\
\hline$\leq 5$ versus $\geq 6 \mathrm{~mm}$ & $\leq 50 \%$ versus $>50 \%$ & 75.5 & 9 & 16 & 15 & 60 & 0.39 \\
\hline Reference WBC & Test BANA & Accuracy (\%) & FP (\%) & FN (\%) & TP $(\%)$ & TN (\%) & Cohen \\
\hline $0 \%$ versus $>0 \%$ & $0 \%$ versus $>0 \%$ & 61.7 & 8 & 30 & 35 & 27 & 0.27 \\
\hline $0 \%$ versus $>0 \%$ & $\leq 50 \%$ versus $>50 \%$ & 53.8 & 3 & 44 & 22 & 32 & 0.20 \\
\hline$\leq 50 \%$ versus $>50 \%$ & $0 \%$ versus $>0 \%$ & 64 & 12 & 24 & 30 & 34 & 0.29 \\
\hline$\leq 50 \%$ versus $>50 \%$ & $\leq 50 \%$ versus $>50 \%$ & 61.9 & 4 & 34 & 20 & 42 & 0.27 \\
\hline
\end{tabular}

All comparisons are significant at $P<0.001$.

* Best agreement.

\section{DISCUSSION}

The great advantage of using probing depths as a means of diagnosing periodontal disease is the speed of execution and immediacy in interpretation. In the present investigation, the measurement and recording of probing depths around four representative teeth took about 1 minute. This was less than the 2 minutes that it took to obtain the plaque samples used to prepare microscopic slides and the BANA test. Another 3 minutes was used to analyze the samples for the microscopic count, whereas the BANA reading took no additional effort and was available for interpretation 5 minutes later. But if the microscopic counting was limited to WBCs, then the entire process from sampling to counting would take about 3 minutes. If only the BANA test was used, then the processing would take about 1 to 2 minutes. These considerations would indicate that the time advantage enjoyed by using probing depths is not so great as to preclude the use of other diagnostic criteria if these criteria can be demonstrated to be more accurate in diagnosing periodontal disease/infections.

The use of probing depths to diagnose periodontal disease is a surrogate for more meaningful measurements such as bone and attachment loss. As such, it is a one-dimensional measurement, albeit the most important dimension, of the volume of the space, or pocket, overlying an area on the root surface of a tooth, and has a large measurement error $( \pm 1 \mathrm{~mm})$ so that the measuring error for a $5-\mathrm{mm}$ pocket would be $40 \%$, i.e., 
Table 4.

\section{Comparison of Probing Depths $\geq 5 \mathrm{~mm}$ and WBC Levels $>50 \%$ of Sites as a Guide in the Recommendation of Periodontal Treatment for Subjects}

\begin{tabular}{|c|c|c|c|c|}
\hline \multirow[b]{2}{*}{ WBC (test) } & \multirow[b]{2}{*}{$\begin{array}{c}\text { Clinical } \\
\text { Recommendation }\end{array}$} & \multicolumn{2}{|c|}{$\begin{array}{c}\text { Probing } \\
\text { Depth (reference) }\end{array}$} & \\
\hline & & $\begin{array}{c}\leq 4 \mathrm{~mm} \\
\text { No Treatment }\end{array}$ & $\begin{array}{c}\geq 5 \mathrm{~mm} \\
\text { Treatment }\end{array}$ & \\
\hline $\begin{array}{c}\leq 50 \% \text { sites } \\
\text { positive }\end{array}$ & No treatment & 350 & 130 & 480 \\
\hline \multirow{2}{*}{$\begin{array}{c}>50 \% \text { sites } \\
\text { positive }\end{array}$} & Treatment & 143 & 420 & 563 \\
\hline & & 493 & 550 & 1,043 \\
\hline
\end{tabular}

If probing depth is the reference standard, then sensitivity $=76.4 \%$; specificity $=71 \%$; accuracy $=73.8 \%$; positive predictive value $=74.6 \%$; negative predictive value $=72.9 \%$; false negative $=130$; and false positive $=$ 143. If $\mathrm{WBC}$ is the reference standard, then $76.4 \%=$ positive predictive value; $71 \%=$ negative predictive value; $73.8 \%=$ accuracy; $74.6 \%=$ sensitivity; $72.9 \%=$ specificity; $130=$ false positive; and $143=$ false negative.

$2 / 5 \mathrm{~mm} .{ }^{19}$ This means that some teeth (subjects) with no disease/infection may be incorrectly recommended for treatment, whereas others with disease/infection would not be recommended for treatment based on measuring error alone. These considerations indicate that an alternate means of diagnosing periodontal disease based upon indicators of an inflammatory process/infection should be pursued.

Probing depth was considered to be the primary standard in the chi-square $\left(\chi^{2}\right)$ comparisons with the percentage of sites WBC positive or percentage of sites BANA positive because it is the de facto clinical standard. At each threshold for probing depths, the percentage of sites WBC positive or percentage of sites BANA positive were significantly related to probing depths $(P<0.0001$; Table 3$)$ in their ability to recognize patients with periodontal disease. But the percentage of accuracy indicated that these diagnostic criteria were identifying the same patients in $60 \%$ to $75 \%$ of the comparisons.

This result was illustrated by analyzing the number of patients who would be recommended for treatment when the threshold for probing depths was $\geq 5 \mathrm{~mm}$ and that for the percentage of WBCs was $>50 \%$ of sites (Table 4). This probing depth threshold would have recommended periodontal treatment for 550 patients. Seventy-six percent of these patients would have received the same recommendation if the threshold of $>50 \%$ WBCs was used, but the remaining $24 \%$, or 130 patients, who had $\leq 50 \%$ of sites that were WBC positive, would also have been recommended for treatment. If the comparison was between probing depths $\geq 5 \mathrm{~mm}$ and percentage of WBCs $>0 \%$, then $8 \%$ or 87 patients without any evidence of inflammatory disease would have been recommended for treatment (calculated from date presented in Table 3). Thus, appreciable numbers of patients without evidence of inflammatory disease would have been diagnosed by probing depths as having a disease that is inflammatory in nature.

If the roles were reversed, with the threshold of $>50 \%$ WBCs used as the primary decision for recommending periodontal treatment, then 563 patients would have been recommended for treatment, $74.6 \%$ of whom would also have been identified by the probing depth threshold (Table 4). This means that $25.6 \%$, or 143 patients, with probing depths $\leq 4 \mathrm{~mm}$, would have been recommended for treatment because of the presence of inflammatory markers in the plaque sample.

These differences in treatment recommendations raised the question, "Is it better to treat sites (patients) with deep pockets without much evidence of inflammatory markers or to treat sites (patients) with inflammatory markers but without deep pockets? Insights into this question were sought with multivariate diagnostic models.

All diagnostic models were highly significant and shared certain predictors in common (Table 5). For instance, probing depth, as an indicator of bacteria mass, was a significant predictor of periodontal disease in the WBC and BANA models. The percentage of WBC-positive sites was an even stronger predictor of periodontal disease in the probing depth model $(\mathrm{OR}=2.8)$ and the BANA model $(\mathrm{OR}=2.1)$. The percentage of BANA-positive sites was a very strong predictor in the probing depth model $(\mathrm{OR}=5.3)$ and in the WBC model $(\mathrm{OR}=2.1)$ The PBS was a strong predictor of periodontal disease in the probing depth model $(\mathrm{OR}=2.6)$ and the WBC model $(\mathrm{OR}=2.2)$.

The WBC model had an ROC curve of $90 \%$, which is very high, and was unique in having microbial variables as important predictors of treatment needs, i.e., the percentage of small gliding rods $(\mathrm{OR}=6.3$ ), percentage of spinning rods $(\mathrm{OR}=4.9)$, and percentage of spirochetes $(O R=1.9)$. The identity of the spinning and gliding rods is not known, but they would be categorized as motile rods, a group that has repeatedly been associated with periodontal disease by semiquantitative darkfield-microscopy examinations of plaque samples ${ }^{11,12,20-22}$ and by qualitative phasecontrast microscopy. ${ }^{10}$ The fact that the spinning rods were also a significant predictor of treatment needs in the BANA model would suggest that they may be the single most important bacterial determinant of periodontal disease/infections, and they remained to be identified at the species level. 
Table 5.

\section{Comparison of Models in Their Ability to Diagnose Periodontal Disease}

\begin{tabular}{|c|c|c|c|c|c|c|}
\hline & \multicolumn{2}{|c|}{ Probing Model $\geq 5 \mathrm{~mm}$} & \multicolumn{2}{|c|}{$\begin{array}{l}\text { WBC Model }>50 \% \\
\text { Positive Sites }\end{array}$} & \multicolumn{2}{|c|}{$\begin{array}{c}\text { BANA Model }>0 \% \\
\text { Positive Sites }\end{array}$} \\
\hline AIC statistic & \multicolumn{2}{|c|}{908} & \multicolumn{2}{|c|}{826} & \multicolumn{2}{|c|}{1226} \\
\hline ROC curve & \multicolumn{2}{|c|}{87.4} & \multicolumn{2}{|c|}{90.4} & \multicolumn{2}{|c|}{73.7} \\
\hline Predictor & $P$ & OR & $P$ & OR & $P$ & OR \\
\hline Variable: & & & & & & \\
\hline Age & $<0.001$ & 1.03 & & & 0.015 & 1.01 \\
\hline Smoker never & & & & & & \\
\hline Quit & $<0.001$ & 2.72 & & & 0.068 & 1.34 \\
\hline Current & 0.001 & 2.36 & & & 0.78 & 0.94 \\
\hline Probing depth & & NA & 0.002 & 1.3 & $<0.001$ & 1.34 \\
\hline PBS & $<0.001$ & 2.56 & $<0.001$ & 2.23 & & \\
\hline WBC positive (\%) & $<0.001$ & 2.76 & & NA & 0.001 & 2.09 \\
\hline BANA positive (\%) & $<0.001$ & 5.26 & 0.008 & 2.09 & & NA \\
\hline Small gliding rods (\%) & & & $<0.001$ & 6.27 & & \\
\hline Spinning rods (\%) & & & $<0.001$ & 4.91 & $<0.022$ & 1.69 \\
\hline Spirochetes (\%) & 0.024 & 1.90 & 0.06 & 1.86 & & \\
\hline Likelihood ratio & & 502.7 & & 627.3 & & 164. \\
\hline Degrees of freedom & & 7 & & 6 & & 5 \\
\hline
\end{tabular}

All models are significant at $P<0.0001$. The best score for each test is in bold type.

$\mathrm{AIC}=-2 \log (\mathrm{L})+2 \mathrm{p}$ where $\mathrm{L}$ is the likelihood statistics and $\mathrm{p}$ is the number of predictors in the model.

A low AIC value is better, which indicates a better fit (high $\mathrm{L}$ ) with fewer degrees of freedom (low $\mathrm{p}$ ).

$\mathrm{NA}=$ a variable is not applicable in the model under consideration.

$\mathrm{ROC}=$ receiver operator curve, where the higher the score, the larger the area under the curve.

The probing depth model demonstrated the validity of the statistical approaches that were used as this model captured an eclectic mix of demographic, clinical, microscopic, and bacteriological findings that have been historically associated with periodontal disease. For example, increasing age had a slight but significant effect on the diagnosis of periodontal disease, which is consistent with epidemiological studies that have associated age with periodontal disease. ${ }^{23,24} \mathrm{~A}$ history of smoking and current smoking were strong predictors of periodontal disease. ${ }^{25,26}$ Gingivitis, as measured with the PBS, was also a strong predictor, which is consistent with many studies that have emphasized bleeding as a diagnostic indicator of periodontal disease. 8,27 The probing depth model also had strong inflammatory and infection components, as the percentage of WBC-positive sites, percentage of BANA-positive sites, and percentage of spirochete-positive sites were strong predictors of treatment needs. Keyes and Rams ${ }^{10}$ have implicated the presence of WBCs in periodontal disease, and many studies have linked spirochetes to periodontal disease. ${ }^{28,29}$ The BANA test has been associated with periodontal disease in epidemiologi$\mathrm{cal}^{30,31}$ and clinical studies. ${ }^{32}$

When the diagnostic model was based upon the presence of WBCs, traditional risk determinants, such as age and smoking status, were not significant. As the pathophysiology in periodontal disease is an inflammatory response to a bacterial overgrowth of certain bacteria in the plaque, age, per se, should have no obvious effect on such an infection. Smoking has been shown to be a risk factor when probing depths have been used, ${ }^{24}$ but its role, other than decreasing the blood flow to the gingiva, ${ }^{33}$ has not been elucidated. Several studies have shown that current smokers and former smokers have higher percentages of BANA-positive plaques $^{34}$ and higher levels of BANA-positive species such as $P$. gingivalis, $T$. forsythensis, and $T$. denticola in their plaques. $^{35,36}$ This raises the possibility that the contributions made by the BANA results and smoking status to the clinical outcome in the WBC model were so correlated that only one, the percentage BANA positive sites, emerged as a significant predictor for the diagnosis. This suggests that smoking could have been a surrogate for an infection due to BANA-positive species in those studies that identified smoking as a risk factor for periodontal disease.

The BANA model showed a modest effect of age $(\mathrm{OR}=1.01)$ and probing depth $(\mathrm{OR}=1.34)$ but was more dependent on the percentage of sites positive for WBC $(O R=2.09)$ and the percentage of sites positive for spinning rods $(\mathrm{OR}=1.69)$ as significant predictors of subjects being diagnosed with periodontal disease. The absence of spirochetes was unexpected, as previous studies have implicated spirochetal counts with a positive BANA test. ${ }^{37}$ 
The detection of WBCs in a plaque sample would seem to capture the host response to all of the periodontopathogens, such as spinning rods, small gliding rods, spirochetes, and BANA-positive species, as well as the bacterial mass reflected by the probing depth and the bleeding that occurs as the tissue response to the infection. This superiority of WBCs as a diagnostic for periodontal disease can be transferred to the clinical setting. With some training, clinicians could confidently use phase-contrast microscopic analysis of WBC levels in plaque to place their patients into risk categories of periodontal disease or health. This approach would change the diagnosis from periodontal disease to periodontitis and would be in harmony with the pathophysiology of the disease process. It would also emphasize the expanding recognition of the contributions of inflammatory diseases, including periodontitis, to systemic health such as cardiovascular disease and preterm births. ${ }^{38,39}$

\section{CONCLUSION}

These results indicate that periodontal disease can be diagnosed chairside by the presence of WBCs in plaque samples, a finding that reflects the inflammatory nature of the disease process.

\section{ACKNOWLEDGMENT}

Support for this study was a gift from Oralife, Toronto, Ontario. Several patents on the BANA test have been assigned to Dr. Loesche. Dr. Loesche is a partner in BANAMet, Ann Arbor, MI, which supplied BANA incubators.

\section{REFERENCES}

1. Oliver RC, Brown LJ, Löe H. Periodontal diseases in the United States population. J Periodontol 1998;69: 269-278.

2. Albandar JM, Brunelle JA, Kingman A. Destructive periodontal disease in adults 30 years of age and older in the United States, 1988-1994. J Periodontol 1999; 70:13-29.

3. Papapanou PN. Periodontal diseases: Epidemiology. Ann Periodontol 1996;1:1-36.

4. Loesche WJ, Grossman NS. Periodontal disease as a specific, albeit chronic, infection: Diagnosis and treatment. Clin Microbiol Rev 2001;14:727-752.

5. American Academy of Periodontology. Oral health information for the public: Periodontal (gum) diseases. Available at: http://www.perio.org/consumer/2a.html. Accessed April 12, 2006.

6. Genco RJ, Goldman HM, Cohen DW. Contemporary Periodontics, 1st ed. St. Louis, MO: C.V. Mosby; 1990.

7. Lindhe J, Karring T, Lang N. Clinical Periodontology and Implant Dentistry, 3rd ed. Copenhagen: Munksgaard; 1998.

8. Newbrun E. Indices to measure gingival bleeding. $J$ Periodontol 1996;67:555-561.

9. Loesche W, Laughon B. Role of spirochetes in periodontal disease. In: Genco RJ, Mergenhagen SE, eds.
Host-Parasite Interactions in Periodontal Disease. Washington, DC: American Society for Microbiology; 1982:62-80

10. Keyes PH, Rams TE. A rationale for management of periodontal diseases: Rapid identification of microbial 'therapeutic targets' with phase-contrast microscopy. J Am Dent Assoc 1983;106:803-812.

11. Keyes PH, Rams TE. Clinical applications of microbiologically monitored and modulated periodontal therapy. N Y State Dent J 1983;49:478-481.

12. Loesche WJ, Syed SA, Schmidt E, Morrison EC. Bacterial profiles of subgingival plaques in periodontitis. J Periodontol 1985;56:447-456.

13. Socransky SS, Haffajee AD, Cugini MA, Smith C, Kent RL Jr. Microbial complexes in subgingival plaque. $J$ Clin Periodontol 1998;25:134-144.

14. Socransky SS, Smith C, Martin L, Paster BJ, Dewhirst FE, Levin AE. "Checkerboard" DNA-DNA hybridization. Biotechniques 1994;17:788-792.

15. Loesche WJ, Bretz WA, Kerschensteiner D, et al. Development of a diagnostic test for anaerobic periodontal infections based on plaque hydrolysis of benzoyl-DL-arginine-naphthylamide. J Clin Microbiol 1990;28:1551-1559.

16. Loesche WJ, Lopatin DE, Giordano J, Alcoforado G, Hujoel P. Comparison of the benzoyl-DL-argininenaphthylamide (BANA) test, DNA probes, and immunological reagents for ability to detect anaerobic periodontal infections due to Porphyromonas gingivalis, Treponema denticola, and Bacteroides forsythus. $J$ Clin Microbiol 1992;30:427-433.

17. Takaishi Y, Morii H, Miki T. The benzoyl-DL argininenaphthylamide (BANA) test and polymerase chain reaction measurement of pathogenic bacteria can assess the severity of periodontal disease. Int $J$ Tissue React 2003;25:19-24.

18. Loesche WJ. Clinical and microbiological aspects of chemotherapeutic agents used according to the specific plaque hypothesis. J Dent Res 1979;58:2404-2412.

19. Loesche WJ. The antimicrobial treatment of periodontal disease: Changing the treatment paradigm. Crit Rev Oral Biol Med 1999;10:245-275.

20. Listgarten MA, Hellden L. Relative distribution of bacteria at clinically healthy and periodontally diseased sites in humans. J Clin Periodontol 1978;5: $115-132$.

21. Lindhe J, Liljenberg B, Listgarten M. Some microbiological and histopathological features of periodontal disease in man. J Periodontol 1980;51:264-269.

22. Wolff LF, Pihlstrom BL, Liljemark WF, Schaffer EM, Bandt CL. Distinct categories of microbial forms associated with periodontal disease. J Periodontal Res 1985;20:497-502.

23. Löe H, Anerud A, Boysen H. The natural history of periodontal disease in man: Prevalence, severity, and extent of gingival recession. J Periodontol 1992;63: 489-495

24. Grossi SG, Zambon JJ, Ho AW, et al. Assessment of risk for periodontal disease. I. Risk indicators for attachment loss. J Periodontol 1994;65:260-267.

25. Beck JD, Slade GD. Epidemiology of periodontal diseases. Curr Opin Periodontol 1996;3:3-9.

26. Bergstrom J. Tobacco smoking and chronic destructive periodontal disease. Odontology 2004;92:1-8.

27. Lang NP, Joss A, Tonetti MS. Monitoring disease during supportive periodontal treatment by bleeding on probing. Periodontol 2000 1996;12:44-48. 
28. Loesche WJ. The role of spirochetes in periodontal disease. Adv Dent Res 1988;2:275-283.

29. Ellen RP, Galimanas VB. Spirochetes at the forefront of periodontal infections. Periodontol 2000 2005;38: 13-32.

30. Bretz WA, Eklund SA, Radicchi R, et al. The use of a rapid enzymatic assay in the field for the detection of infections associated with adult periodontitis. J Public Health Dent 1993;53:235-240.

31. Okawa Y, Takahashi Y, Muramatsu J, Ishii T. Sitespecific CPITN score and periodontal disease activity assessed by the paper BANA assay. Bull Tokyo Dent Coll 1993;34:173-176.

32. Loesche WJ, Bretz WA, Lopatin D, et al. Multi-center clinical evaluation of a chairside method for detecting certain periodontopathic bacteria in periodontal disease. J Periodontol 1990;61:189-196.

33. Bergström J, Persson L, Preber H. Influence of cigarette smoking on vascular reaction during experimental gingivitis. Scand J Dent Res 1988;96:34-39.

34. Kazor C, Taylor GW, Loesche WJ. The prevalence of BANA-hydrolyzing periodontopathic bacteria in smokers. J Clin Periodontol 1999;26:814-821.
35. Zambon JJ, Grossi SG, Machtei EE, Ho AW, Dunford $\mathrm{R}$, Genco RJ. Cigarette smoking increases the risk for subgingival infection with periodontal pathogens. $J$ Periodontol 1996;67(Suppl.):1050-1054.

36. Haffajee AD, Socransky SS. Relationship of cigarette smoking to the subgingival microbiota. J Clin Periodontol 2001;28:377-388.

37. Loesche WJ, Giordano J, Hujoel PP. The utility of the BANA test for monitoring anaerobic infections due to spirochetes (Treponema denticola) in periodontal disease. J Dent Res 1990;69:1696-1702.

38. Loesche WJ. Anaerobic periodontal infections as risk factors for medical diseases. Curr Infect Dis Rep 1999; $1: 33-38$.

39. Offenbacher S, Beck JD. A perspective on the potential cardioprotective benefits of periodontal therapy. Am Heart J 2005; 149:950-954.

Correspondence: Dr. David Apsey, 31020 Utica Rd., Fraser, MI 48026. Fax: 586/293-5990.

Accepted for publication April 26, 2006. 